\section{BRAZIULIAN JOURNAL}

OF MEDICAL AND BIOLOGICAL RESFARCH

www.bjournal.com.br
ISSN 0100-879X

Volume 43 (02) 124-225 February 2010

BIOMEDICAL SCIENCES

AND

CLINICAL INVESTIGATION

Braz J Med Biol Res, February 2010, Volume 43(2) 211-216

Acute electrophysiologic consequences of pyridostigmine inhibition of cholinesterase in humans

L.I. Zimerman, A. Liberman, R.R.T. Castro, J.P. Ribeiro and A.C.L. Nóbrega

The Brazilian Journal of Medical and Biological Research is partially financed by
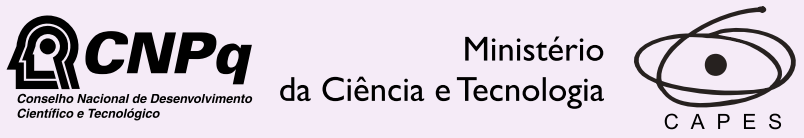

Ministério da Educação

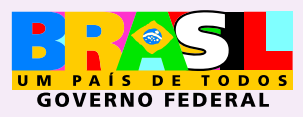

Institutional Sponsors 


\title{
Acute electrophysiologic consequences of pyridostigmine inhibition of cholinesterase in humans
}

\author{
L.I. Zimerman ${ }^{1,2}$, A. Liberman ${ }^{1}$, R.R.T. Castro ${ }^{3}$, J.P. Ribeiro ${ }^{1,2}$ \\ and A.C.L. Nóbrega ${ }^{3,4}$ \\ ${ }^{1}$ Serviço de Cardiologia, Hospital de Clínicas de Porto Alegre, Porto Alegre, RS, Brasil \\ 2Departamento de Medicina, Faculdade de Medicina, Universidade Federal do Rio Grande do Sul, \\ Porto Alegre, RS, Brasil \\ ${ }^{3}$ Departamento de Fisiologia e Farmacologia, ${ }^{4}$ Pós-graduação em Ciências Cardiovasculares, \\ Universidade Federal Fluminense, Niterói, RJ, Brasil
}

\begin{abstract}
The cardiovascular electrophysiologic basis for the action of pyridostigmine, an acetylcholinesterase inhibitor, has not been investigated. The objective of the present study was to determine the cardiac electrophysiologic effects of a single dose of pyridostigmine bromide in an open-label, quasi-experimental protocol. Fifteen patients who had been indicated for diagnostic cardiac electrophysiologic study underwent two studies just before and 90-120 min after the oral administration of pyridostigmine $(45 \mathrm{mg})$. Pyridostigmine was well tolerated by all patients. Wenckebach nodal anterograde atrioventricular point and basic cycle were not altered by pyridostigmine. Sinus recovery time (ms) was shorter during a 500-ms cycle stimulation (pre: $326 \pm 45$ vs post: $235 \pm 47 ; \mathrm{P}=0.003$ ) but not during $400-\mathrm{ms}$ (pre: $275 \pm 28$ vs post: $248 \pm 32 ; \mathrm{P}=0.490$ ) or $600-\mathrm{ms}$ (pre: 252 \pm 42 vs post: $179 \pm 26 ; \mathrm{P}=0.080)$ cycle stimulation. Pyridostigmine increased the ventricular refractory period (ms) during the 400 -ms cycle stimulation (pre: $238 \pm 7$ vs post: $245 \pm 9$; $\mathrm{P}=0.028$ ) but not during the 500 -ms (pre: $248 \pm 7$ vs post: $253 \pm$ 9; $P=0.150$ ) or $600-m s$ (pre: $254 \pm 8$ vs post: $259 \pm 8 ; P=0.255$ ) cycle stimulation. We conclude that pyridostigmine did not produce conduction disturbances and, indeed, increased the ventricular refractory period at higher heart rates. While the effect explains previous results showing the anti-arrhythmic action of pyridostigmine, the clinical impact on long-term outcomes requires further investigation.
\end{abstract}

Key words: Cardiac electrophysiology; Parasympathetic nervous system; Cholinesterase inhibitors; Cardiovascular disease; Pyridostigmine bromide; Autonomic nervous system

\section{Introduction}

Autonomic dysfunction is a strong and independent predictor of the development of lethal cardiac arrhythmias and sudden death in patients with heart disease (1-4). The concept that adrenergic hyperactivity could be deleterious has led to the widespread clinical use of beta-blockers in patients after acute myocardial infarction (5), with a consequent reduction of overall mortality in these patients (6), as well as in those with heart failure (7). Conversely, few studies have investigated therapeutic alternatives against parasympathetic dysfunction (8), despite the fact that decreased parasympathetic activity represents an independent risk factor in patients after acute myocardial infarction $(9,10)$.
Pyridostigmine, a reversible anticholinesterase agent, i.e., a drug with an indirect vagomimetic effect, seems to be potentially useful in this context. Pyridostigmine has been traditionally used for the treatment of myasthenia gravis due to its action on the motor plate in skeletal muscle (11). In addition, it has been shown to counteract the adverse anti-cholinergic effects of disopyramide, such as decreased baseline saliva and tear production (12). We have previously shown that short-term administration of pyridostigmine may be also potentially useful for cardiovascular protection since in healthy individuals and patients with cardiovascular diseases it results in beneficial effects on markers of cardiovascular risk and dysfunction (8,13-19).

Correspondence: J.P. Ribeiro, Serviço de Cardiologia, Hospital de Clínicas de Porto Alegre, Rua Ramiro Barcelos, 2350, 90035-007 Porto Alegre, RS, Brasil. Fax: +55-51-316-8657. E-mail: jpribeiro@cpovo.net

Received April 9, 2009. Accepted November 4, 2009. Available online January 15, 2010. Published February 1, 2010. 
In patients with coronary artery disease, pyridostigmine improves the autonomic and hemodynamic responses to exercise $(13,14,19)$, prevents the myocardial dysfunction induced by mental stress (16), and reduces QTc dispersion (18). In a randomized, crossover, placebo-controlled, and double-blind study, pyridostigmine administration increased heart rate variability and reduced ventricular arrhythmia density in patients with heart failure (17), an effect mediated, at least in part, by stimulation of the endogenous nitric oxide pathway (20). More recently (19), it was shown that pyridostigmine improves the hemodynamic profile of heart failure patients during dynamic exercise.

Despite the effects of pyridostigmine on these surrogate endpoints (21), the electrophysiologic basis for its action has not been investigated. Since pyridostigmine is a cholinomimetic drug, it is expected to reduce sinusal automatism (22) and chronotropic and dromotropic function (23), increasing the cardiac excitability threshold (24) and the refractory period of sinusal and atrioventricular nodes, but reducing the atrial refractory period $(25,26)$. Therefore, the purpose of the present study was to determine the cardiac electrophysiologic consequences of a single oral dose of pyridostigmine bromide in man.

\section{Material and Methods}

\section{Patients}

Fifteen consecutive patients referred to our service by their attending physician for diagnostic cardiac electrophysiologic study were included in the present investigation. The clinical characteristics of these patients are shown in Table 1. All patients continued to use their current medication during the study. Exclusion criteria were bradyarrhythmias, implanted pacemaker, pregnancy, previous use of or allergy to pyridostigmine, bowel dysfunction, urinary retention, and the indication of isoproterenol infusion during the electrophysiologic study. The protocol conformed to the principles outlined in the Declaration of Helsinki and was approved by the Research Ethics Committee on Human Research of the Universidade Federal do Rio Grande do Sul. All patients gave written informed consent to participate in the study after full explanation of the procedures and their potential risks.

\section{Protocol}

Following an open-label, quasi-experimental protocol, a single dose of pyridostigmine $(45 \mathrm{mg})$ was orally administered to all patients just before the electrophysiologic study. Ninety to 120 min after administration of the drug, another electrophysiologic study was performed. Both studies were performed under sedation (iv midazolam in incremental doses until sedation). Patients were continuously monitored with a chest electrocardiogram and for oxygen saturation and noninvasive blood pressure determination during the study. Patients remained in the hospital for the next $6 \mathrm{~h}$ following the study, so that any side effect of the drug could be recorded.

\section{Electrophysiologic study}

The electrophysiologic study was performed as described (27). Under fluoroscopic guidance, multipolar electrode catheters were positioned against the upper right atrial wall, near the region of the sinus node, and across the tricuspid valve in the area of the His bundle for pacing and recording of intracardiac electrograms. Basic intracardiac conduction intervals were then measured during sinus rhythm (28). Programmed electrical stimulation of the atrium was performed (UHS 20, Biotronik, Germany) using 2-ms constant current pulses at approximately twice the stimulation threshold. Surface ECG leads and bipolar intracardiac electrograms filtered at a bandpass of 30 to $500 \mathrm{~Hz}$ were displayed and recorded simultaneously on a multichannel recorder (Mingograf 7, Siemens, Germany). After control recordings, the atrium was paced during 30-s periods at constant cycle lengths of 600,500 and $400 \mathrm{~ms}$ and sinus node recovery time was measured. The AV nodal Wenckebach cycle was defined as the length of the cycle during incremental atrial pacing in which the AV nodal Wenckebach phenomenon was noted. The anterograde

Table 1. Clinical characteristics of the 15 patients.

\begin{tabular}{ll}
\hline Age (years) & $56 \pm 4$ \\
Males & $8(53 \%)$ \\
Body mass index $\left(\mathrm{kg} / \mathrm{m}^{2}\right)$ & $26 \pm 2$ \\
Indication for the electrophysiologic study & \\
Syncope & $9(60 \%)$ \\
Palpitations & $5(33 \%)$ \\
Risk stratification for sudden death & $1(7 \%)$ \\
Comorbidities & \\
Coronary artery disease & $4(27 \%)$ \\
Heart failure & $4(27 \%)$ \\
Hypertension & $2(13 \%)$ \\
Chagas' disease & $1(7 \%)$ \\
No known comorbidities & $7(46 \%)$ \\
Medications & \\
Amiodarone & $2(13 \%)$ \\
Diuretics & $4(27 \%)$ \\
Angiotensin-converting enzyme & $7(46 \%)$ \\
Calcium channel blockers & $2(13 \%)$ \\
Beta-blockers & $4(27 \%)$ \\
Acetylsalicylic acid & $4(27 \%)$ \\
Nitrates & $1(7 \%)$ \\
Digoxin & $1(7 \%)$ \\
Statins & $3(20 \%)$ \\
\hline
\end{tabular}

Data are reported as means \pm SEM or number of patients with percent in parentheses. 
effective refractory period of the AV node was measured using an 8-beat drive at a cycle length equal to the sinus cycle length minus $100 \mathrm{~ms}$ followed by single premature atrial stimuli introduced decrementally at $10-\mathrm{ms}$ intervals. The ventricular refractory period was measured with the introduction of extrastimuli in 10-ms decrements after an 8-beat drive at cycle lengths of 600,500 , and $400 \mathrm{~ms}$.

\section{Statistical analysis}

Descriptive data are reported as means \pm SEM. Based on the data of Wit et al. (22), a sample size of 14 patients was required to detect a $20 \%$ difference in the refractory ventricular period, with an alpha error of 0.01 and power of 0.80 . Electrophysiologic variables obtained in the first study without the effect of pyridostigmine (pre) were compared to those measured in the second study under the action of pyridostigmine (post) using the two-tailed paired Student $t$-test or the Wilcoxon test, when appropriate. Significance was set at $P<0.05$.

\section{Results}

Pyridostigmine was well tolerated by all patients. Table 2 presents the electrophysiologic data before and after pyridostigmine administration. Wenckebach nodal anterograde atrioventricular point and basic cycle were not altered by pyridostigmine. The sinus recovery time was shorter during the 500 $\mathrm{ms}$ cycle stimulation but not during 400- or 600-ms cycle stimulation. PA, AH, and HV intervals presented normal values and were not significantly different after pyridostigmine. Pyridostigmine administration significantly increased the ventricular refractory period during the 400-ms cycle stimulation but not during the 500- or 600-ms cycle stimulation (Figure 1). Data from patients who were taking beta-blockers or amiodarone were analyzed separately and showed similar results for sinus recovery time during the 500-ms cycle stimulation (pre: $263 \pm 74$ vs post: $157 \pm 61 \mathrm{~ms} ; \mathrm{P}=0.04$ ) and a ventricular refractory period during the 400-ms cycle stimulation (pre: $242 \pm 15$ vs $253 \pm 18 \mathrm{~ms}$; $\mathrm{P}=0.03)$.

\section{Discussion}

Pyridostigmine is an acetylcholinesterase
Table 2. Electrophysiologic effects of pyridostigmine.

\begin{tabular}{lcc}
\hline & Pre-pyridostigmine & Post-pyridostigmine \\
\hline Basic cardiac cycle (ms) & $906 \pm 37$ & $914 \pm 39$ \\
Wenckebach nodal anterograde & $400 \pm 20$ & $398 \pm 17$ \\
$\quad$ ventricular point (ms) & & \\
Sinus node recovery time (ms) & $275 \pm 28$ & $248 \pm 32$ \\
$\quad 400$-ms cycle stimulation & $326 \pm 45$ & $235 \pm 47^{*}$ \\
500 -ms cycle stimulation & $252 \pm 42$ & $179 \pm 26$ \\
600 -ms cycle stimulation & $36 \pm 2$ & $36 \pm 1$ \\
PA interval (ms) & $88 \pm 8$ & $93 \pm 9$ \\
AH interval (ms) & $48 \pm 3$ & $50 \pm 2$ \\
HV interval (ms) & $147 \pm 32$ & $123 \pm 31$ \\
Atrial refractory period (ms) & $147 \pm 32$ & $123 \pm 31$ \\
500 -ms cycle stimulation & & \\
600 -ms cycle stimulation & $323 \pm 16$ & $316 \pm 20$ \\
Atrioventricular refractory period (ms) & $327 \pm 20$ & $345 \pm 23$ \\
500 -ms cycle stimulation & & \\
600 -ms cycle stimulation & $238 \pm 7$ & $245 \pm 9^{*}$ \\
Ventricular refractory period (ms) & $248 \pm 7$ & $253 \pm 9$ \\
400 -ms cycle stimulation & $254 \pm 8$ & $259 \pm 8$ \\
500 -ms cycle stimulation & & \\
600 ms cycle stimulation & & \\
\hline
\end{tabular}

Data are reported as means \pm SEM for 15 patients. Measurements were made before and 90-120 min after receiving $45 \mathrm{mg}$ pyridostigmine orally. ${ }^{*} \mathrm{P}<0.05 \mathrm{com}$ pared to pre-pyridostigmine (two-tailed paired Student $t$-test).

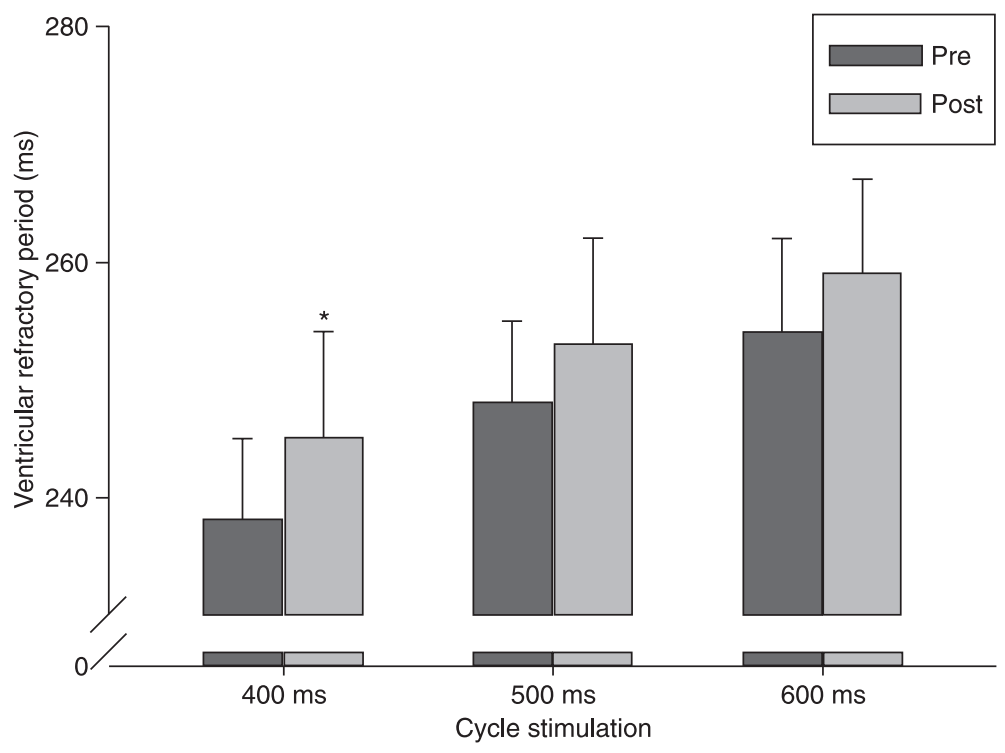

Figure 1. Ventricular refractory periods (means \pm SEM) before and after a single dose of pyridostigmine during stimulation with 400-, 500-, and 600-ms cycles. ${ }^{*} \mathrm{P}<0.05$ compared to the pre-pyridostigmine value (two-tailed paired Student $t$-test). 
reversible inhibitor whose cardiovascular activity has been studied systematically for more than a decade. Studies including healthy volunteers and patients with cardiovascular disease have shown that pyridostigmine shifts the autonomic balance to parasympathetic dominance and may reduce arrhythmia density $(14,17,29)$. A single dose of pyridostigmine elicited bradycardia and reduced QTc dispersion in healthy young subjects (18). When administered to patients with coronary artery disease, pyridostigmine reduced the QTc interval at rest (29) and its recovery from maximal effort (14). Heart rate variability, an established method for analysis of autonomic modulation, was also increased when pyridostigmine was used by healthy volunteers (15) and heart failure patients, reducing ventricular arrhythmia density in the latter (17). The electrophysiologic mechanisms involved in these effects are currently unknown. Despite these promising findings with pyridostigmine, the potential of atrioventricular block, bradycardia leading to hemodynamic instability, and other undesirable parasympathetic effects on cardiac conduction should not be underestimated even when small doses are administered. The present study was undertaken in order to further analyze the mechanistic basis for the potential beneficial and hazardous effects of pyridostigmine. Several previous studies had investigated the autonomic and electrophysiologic properties of another cholinesterase inhibitor, edrophonium hydrochloride. When administered at low doses, edrophonium enhances the parasympathetic drive (30), an effect primarily mediated by autonomic innervations of the heart (31). As a consequence, the atrioventricular conduction and firing rate of the sinoatrial node are inhibited proportionally (32) with no adverse effects (33), and with a reduction of ventricular arrhythmias (34), although inducibility of ventricular fibrillation was not changed in patients with implantable defibrillators (35). Albeit exhibiting promising effects, edrophonium needs to be administered intravenously, precluding its prescription for regular use. Therefore, pyridostigmine, which can be administered orally and causes a dose-dependent vagomimetic effect with few side effects (13-19), is a preferable candidate for the treatment of impaired parasympathetic modulation in heart disease.

As a drug that elicits a parasympathetic effect, pyridostigmine was expected to cause bradycardia in the present study. However, it was quite surprising to observe that the basic cycle was not altered after the use of pyridostigmine. Although cholinesterase activity was not measured in the present protocol, previous studies had shown that even doses smaller than $45 \mathrm{mg}$ were capable of inhibiting cholinesterase serum activity (36). Thus, it is unlikely that a lack of anticholinesterase activity could explain the absence of bradycardia. Sedation could have affected the pharmacodynamics of the drug, altering the time needed for its absorption and for its peak action, but there are no studies that specifically addressed the interactions between midazolam and pyridostigmine. Nevertheless, this is not the first study where bradycardia is not seen after pyridostigmine administration. Indeed, Raj et al. (37) were unable to detect a reduction of heart rate in the seated position $2 \mathrm{~h}$ after pyridostigmine ingestion.

The atrioventricular node is densely innervated with both cholinergic and adrenergic neurons (38) and thus plays an important role in the pathophysiology of arrhythmias, not only protecting ventricles during atrial tachyarrhythmias, but also assuming the pacemaker's role during sinoatrial node failure. The dense cholinergic innervation of the atrioventricular node would make it more susceptible to the occurrence of conduction disturbance secondary to vagomimetic drugs, such as pyridostigmine (39). In the present study, pyridostigmine did not change the Wenckebach point or the atrioventricular conduction intervals, including the $\mathrm{AH}$ interval. A possible explanation is that pyridostigmine, as a systemic drug acting on the heart as a whole, probably acts synchronously on the right and left vagal nerve endings. As previously shown by Schiereck et al. (40), asynchronous right and left stimulation of parasympathetic efferent preganglionic axons causes a greater change in atrioventricular conduction.

The most important finding of the present study is that pyridostigmine increased the ventricular refractory period measured during 400-ms cycle stimulation. This effect was not observed when the ventricular refractory period was studied during longer cycles (500 and $600 \mathrm{~ms}$ ). Therefore, pyridostigmine presents a potential anti-arrhythmic effect at higher heart rates, as occurs during physical exercise and mental stress. Accordingly, previous studies have shown a modulatory action of pyridostigmine on cardiovascular reactivity to exercise and stress, both in healthy subjects (18) and in patients with cardiovascular diseases $(13,14,19)$. Whether adverse outcomes can be hampered by pyridostigmine or the sustained release version of the drug leads to a better effective action with lower side affects remains to be determined.

The results of the present study must be interpreted considering its limitations. Due to obvious ethical reasons, only patients with medical indication for electrophysiologic study were enrolled, composing a heterogeneous group. For the same reasons, there was no difference between the electrophysiologic study performed in volunteers of the present study and the procedure routinely performed at our hospital. A 400-ms period is not routinely used for all measurements during electrophysiologic study in our hospital and that is why data regarding the cycle length of $400 \mathrm{~ms}$ for atrial and AV refractory period were not presented. Also because of ethical limitations, the volunteers were not submitted to a second electrophysiologic study on a different day that would be needed for a placebo-controlled experiment. Nevertheless, a baseline electrophysiologic study was conducted before the administration of pyridostigmine. Considering that pyridostigmine achieves its peak of action at 90-120 $\mathrm{min}$, it is very unlikely that any vagomimetic effect of the 
drug was operating during the first electrophysiologic study. Our data were also obtained under sedation and some of the patients were taking medications that act on the autonomic nervous system. Therefore, baseline sympathetic activity was probably lower than during daily activities. As shown in the Results section, the use of beta-blockers or amiodarone did not modify the results.

These findings not only agree with the previously demonstrated anti-arrhythmic effects of pyridostigmine (17) but also extend the knowledge about pyridostigmine electrophysiologic effects. The oral administration of a

\section{References}

1. La Rovere MT, Bigger JT Jr, Marcus FI, Mortara A, Schwartz PJ. Baroreflex sensitivity and heart-rate variability in prediction of total cardiac mortality after myocardial infarction. ATRAMI (Autonomic Tone and Reflexes After Myocardial Infarction) Investigators. Lancet 1998; 351: 478-484.

2. Schwartz PJ, La Rovere MT. ATRAMI: a mark in the quest for the prognostic value of autonomic markers. Autonomic Tone and Reflexes After Myocardial Infarction. Eur Heart $J$ 1998; 19: 1593-1595.

3. Piepoli MF, Capucci A. Autonomic nervous system in the genesis of arrhythmias in chronic heart failure: implication for risk stratification. Minerva Cardioangiol 2007; 55: 325-333.

4. Hoyer D, Maestri R, La Rovere MT, Pinna GD. Autonomic response to cardiac dysfunction in chronic heart failure: a risk predictor based on autonomic information flow. Pacing Clin Electrophysiol 2008; 31: 214-220.

5. De Luca G, de Boer MJ, Ottervanger JP, van't Hof AW, Hoorntje JC, Gosselink AT, et al. Impact of beta-blocker therapy at discharge on long-term mortality after primary angioplasty for ST-segment elevation myocardial infarction. Am J Cardiol 2005; 96: 806-809.

6. Bigger JT Jr, Coromilas J. How do beta-blockers protect after myocardial infarction? Ann Intern Med 1984; 101: 256-258.

7. Packer M, Bristow MR, Cohn JN, Colucci WS, Fowler MB, Gilbert EM, et al. The effect of carvedilol on morbidity and mortality in patients with chronic heart failure. U.S. Carvedilol Heart Failure Study Group. N Engl J Med 1996; 334: 1349-1355.

8. Nobrega AC, Teixeira De Castro RR. Parasympathetic dysfunction as a risk factor in myocardial infarction: what is the treatment? Am Heart J 2000; 140: E23.

9. Kleiger RE, Miller JP, Bigger JT Jr, Moss AJ. Decreased heart rate variability and its association with increased mortality after acute myocardial infarction. Am J Cardiol 1987; 59: 256-262.

10. Odemuyiwa O, Malik M, Farrell T, Bashir Y, Poloniecki J, Camm J. Comparison of the predictive characteristics of heart rate variability index and left ventricular ejection fraction for all-cause mortality, arrhythmic events and sudden death after acute myocardial infarction. Am J Cardiol 1991; 68: 434-439.

11. Skeie GO, Apostolski S, Evoli A, Gilhus NE, Hart IK, Harms $\mathrm{L}$, et al. Guidelines for the treatment of autoimmune neuromuscular transmission disorders. Eur J Neurol 2006; 13: 691-699. low dose of pyridostigmine was well tolerated and did not produce conduction disturbances. In addition, pyridostigmine increased the ventricular refractory period at higher heart rates.

\section{Acknowledgments}

The authors would like to thank Fundo de Incentivo à Pesquisa do HCPA, CNPq and FAPERJ for financial support.
12. Teichman SL, Ferrick A, Kim SG, Matos JA, Waspe LE, Fisher JD. Disopyramide-pyridostigmine interaction: selective reversal of anticholinergic symptoms with preservation of antiarrhythmic effect. J Am Coll Cardiol 1987; 10: 633641.

13. Castro RR, Porphirio G, Serra SM, Nobrega AC. Cholinergic stimulation with pyridostigmine protects against exercise induced myocardial ischaemia. Heart 2004; 90: 1119-1123.

14. Castro RR, Serra SM, Porphirio G, Mendes FS, Oliveira LP, Nobrega AC. Pyridostigmine reduces QTC interval during recovery from maximal exercise in ischemic heart disease. Int J Cardiol 2006; 107: 138-139.

15. Nobrega AC, dos Reis AF, Moraes RS, Bastos BG, Ferlin EL, Ribeiro JP. Enhancement of heart rate variability by cholinergic stimulation with pyridostigmine in healthy subjects. Clin Auton Res 2001; 11: 11-17.

16. Nobrega AC, Loures DL, Pontes PV, Sant'anna ID, Mesquita ET. Cholinergic stimulation with pyridostigmine prevents the impairment in ventricular function during mental stress in coronary artery disease patients. Int J Cardiol 2008; 125: 418-421.

17. Behling A, Moraes RS, Rohde LE, Ferlin EL, Nobrega AC, Ribeiro JP. Cholinergic stimulation with pyridostigmine reduces ventricular arrhythmia and enhances heart rate variability in heart failure. Am Heart J 2003; 146: 494-500.

18. Castro RR, Serra SM, Nobrega AC. Reduction of QTc interval dispersion. Potential mechanism of cardiac protection of pyridostigmine bromide. Arq Bras Cardiol 2000; 75: 205213.

19. Serra SM, Costa RV, Teixeira De Castro RR, Xavier SS, Nobrega AC. Cholinergic stimulation improves autonomic and hemodynamic profile during dynamic exercise in patients with heart failure. J Card Fail 2009; 15: 124-129.

20. Chowdhary S, Marsh AM, Coote JH, Townend JN. Nitric oxide and cardiac muscarinic control in humans. Hypertension 2004; 43: 1023-1028.

21. van Veldhuisen DJ. Disagreement between drug effects on autonomic function and mortality: another unreliable surrogate endpoint in heart failure? Int J Cardiol 2000; 75: 176-177.

22. Wit AL, Weiss MB, Berkowitz WD, Rosen KM, Steiner C, Damato AN. Patterns of atrioventricular conduction in the human heart. Circ Res 1970; 27: 345-359.

23. Levy MN, Zieske H. Autonomic control of cardiac pacemaker activity and atrioventricular transmission. J Appl Physiol 
1969; 27: 465-470.

24. Markel ML, Miles WM, Zipes DP, Prystowsky EN. Parasympathetic and sympathetic alterations of Mobitz type II heart block. J Am Coll Cardiol 1988; 11: 271-275.

25. Prystowsky EN, Jackman WM, Rinkenberger RL, Heger JJ, Zipes DP. Effect of autonomic blockade on ventricular refractoriness and atrioventricular nodal conduction in humans. Evidence supporting a direct cholinergic action on ventricular muscle refractoriness. Circ Res 1981; 49: 511-518.

26. Prystowsky EN, Naccarelli GV, Jackman WM, Rinkenberger RL, Heger JJ, Zipes DP. Enhanced parasympathetic tone shortens atrial refractoriness in man. Am J Cardiol 1983; 51: 96-100.

27. Stein R, Medeiros CM, Rosito GA, Zimerman LI, Ribeiro JP. Intrinsic sinus and atrioventricular node electrophysiologic adaptations in endurance athletes. J Am Coll Cardiol 2002; 39: 1033-1038.

28. Josephson M. Clinical cardiac electrophysiology: techniques and interpretation. Philadelphia: Lea \& Febiger; 1993.

29. Castro RR, Porphirio G, Serra SM, Nobrega AC. Cholinergic stimulation with pyridostigmine reduces the QTc interval in coronary artery disease. Braz J Med Biol Res 2002; 35: 685689.

30. Deschamps A, Backman SB, Novak V, Plourde G, Fiset P, Chartrand D. Effects of the anticholinesterase edrophonium on spectral analysis of heart rate and blood pressure variability in humans. J Pharmacol Exp Ther 2002; 300: 112117.

31. Stemple DR, Hall RJ, Mason JW, Harrison DC. Electrophysiologic effects of edrophonium in the innervated and the transplanted denervated human heart. Br Heart J 1978; 40: 644-649.

32. Watanabe S, Kono Y, Oishi-Tobinaga Y, Yamada S, Hara M, Kano T. A comparison of the chronotropic and dromotropic actions between adenosine triphosphate and edrophonium in patients undergoing coronary artery bypass graft surgery. J Cardiothorac Vasc Anesth 2002; 16: 598-602.

33. Miller RR, Olson HG, Vera Z, DeMaria AN, Amsterdam EA, Mason DT. Clinical evaluation of the enhancement of vagal tone in acute myocardial infarction by edrophonium hydrochloride: effects on ventricular arrhythmias, His bundle electrography, and left ventricular function. Am Heart J 1977; 93: 222-228.

34. Weiss T, Lattin GM, Engelman K. Vagally mediated suppression of premature ventricular contractions in man. Am Heart J 1975; 89: 700-707.

35. Mitrani RD, Kloosterman EM, Huikuri H, Dylewski J, Atapattu $\mathrm{S}$, Interian A Jr, et al. Muscarinic receptor stimulation with edrophonium hydrochloride does not elevate ventricular fibrillation thresholds in humans. J Cardiovasc Electrophysiol 1999; 10: 809-816.

36. Nobrega AC, Carvalho AC, Bastos BG. Resting and reflex heart rate responses during cholinergic stimulation with pyridostigmine in humans. Braz J Med Biol Res 1996; 29: 1461-1465

37. Raj SR, Black BK, Biaggioni I, Harris PA, Robertson D. Acetylcholinesterase inhibition improves tachycardia in postural tachycardia syndrome. Circulation 2005; 111: 2734-2740.

38. Hucker WJ, Nikolski VP, Efimov IR. Autonomic control and innervation of the atrioventricular junctional pacemaker. Heart Rhythm 2007; 4: 1326-1335.

39. Sampaio KN, Mauad H, Spyer KM, Ford TW. Differential chronotropic and dromotropic responses to focal stimulation of cardiac vagal ganglia in the rat. Exp Physiol 2003; 88: 315-327.

40. Schiereck P, Sanna N, Mosterd WL. AV blocking due to asynchronous vagal stimulation in rats. Am J Physiol Heart Circ Physiol 2000; 278: H67-H73. 